\title{
Long-term survivor diagnosed with arrhythmogenic right ventricular cardiomyopathy/dysplasia
}

\author{
Katsuro Kashima ${ }^{1}$, Yasuhiro Tanaka ${ }^{1}$, Kazuyo Kawabata ${ }^{1}$, Rumiko Eura ${ }^{2}$, \\ Takashi Yamane ${ }^{2}$, Kazuhiko Nakamura ${ }^{1}$, Mitsuharu Nomoto ${ }^{3}$ \\ ${ }^{1}$ Department of Cardiology, National Hospital Organization, Ibusuki Hospital, Kagoshima, Japan \\ ${ }^{2}$ Department of Urology, National Hospital Organization, Ibusuki Hospital, Kagoshima, Japan \\ ${ }^{3}$ Department of Clinical Pathology, National Hospital Organization, \\ Kagoshima Medical Center, Kagoshima, Japan
}

\begin{abstract}
The subject was a 70 year-old man who survived for 31 years after being diagnosed with right ventricular cardiomyopathy, having undergone right ventricular $(R V)$ aneurysmectomy at the age of 39. His arrhythmia and syncopal attacks were effectively abolished after the original aneurysmectomy. Although he frequently suffered from right heart failure, hemodialysis improved his status. However, the patient died due to worsening anasarca caused by RV low output syndrome. Autopsy results indicated extensive replacement of the $R V$ myocardium with fibrous and fatty tissues. This case suggests that patients with arrhythmogenic RV cardiomyopathy, but without left ventricular abnormalities and rapid ventricular arrhythmia, have a relatively favorable prognosis, although $R V$ abnormalities may be progressive. (Cardiol J 2013; 20, 1: 83-86)
\end{abstract}

Key words: right ventricular cardiomyopathy, prognosis, aneurysmectomy

\section{Introduction}

Arrhythmogenic right ventricular cardiomyopathy/dysplasia (ARVC/D) is a heart muscle disorder that can cause life-threatening ventricular arrhythmias, heart failure, and sudden cardiac death [1]. Although several mutations of the gene encoding major desmosomal proteins have been implicated in the pathogenesis of ARVC/D, the rates of progression of the disease and its natural history remain largely unknown $[2,3]$. Herein, we describe the case of a man who survived for 31 years after having been diagnosed with right ventricular (RV) cardiomyopathy.

\section{Case report}

A 70 year-old man was admitted to our hospital because of consciousness disturbance. Thirty- -one years previously, he had been diagnosed with RV cardiomyopathy, and had a surgical resection of the RV free wall, particularly the outflow tract region, which showed a dyskinetic movement and was extensively replaced by fibrous and fatty tissues. The patient complained of several syncopal attacks induced by probable rapid ventricular arrhythmia, which was refractory to control with various antiarrhythmic agents. His arrhythmia and syncopal attacks were effectively abolished after the original aneurysmectomy. The patient was well for a quarter of a century; however, he had been receiving hemodialysis three times per week for the previous seven years due to chronic renal failure. Although he frequently suffered from right heart failure, hemodialysis improved his edematous status.

Fourteen days before admission to our hospital, he had fallen and injured his leg. Although his leg injury had improved following localized treat-

Address for correspondence: Katsuro Kashima, MD, Department of Cardiology, National Hospital Organization,

Ibusuki Hospital, 4145 Juni-cho, Ibusuki City, Kagoshima 891-0498, Japan, tel: +81 99322 2231, fax: +81 993223149 ,

e-mail: k-kashima@ibusuki2.hosp.go.jp

Received: 15.11.2011 Accepted: 13.12.2011 


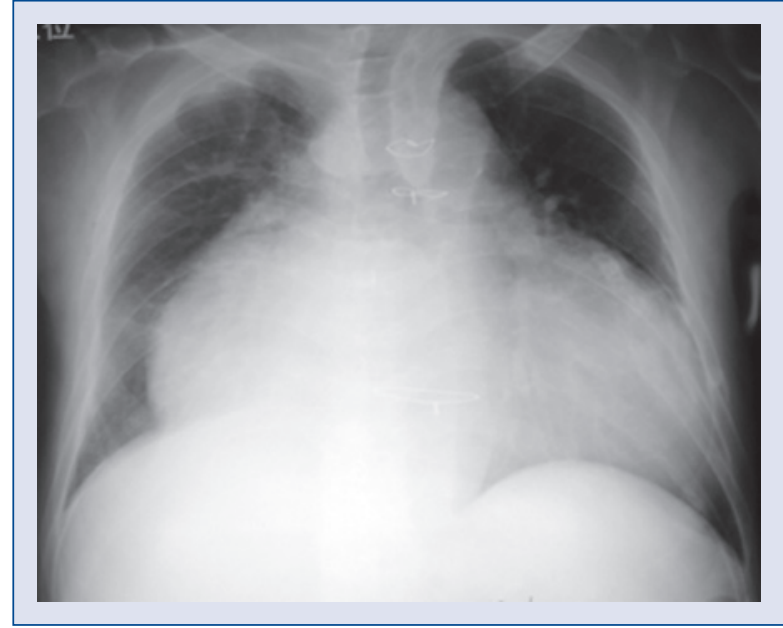

Figure 1. Chest radiography at admission revealed extraordinary cardiomegaly with an $82 \%$ cardiothoracic ratio.

ment, his consciousness level progressively worsened. Chest radiography at admission revealed extraordinary cardiomegaly with an $82 \%$ cardio-thoracic ratio (Fig. 1) compared to $56 \%$ at the original hospitalization 31 years earlier. His electrocardiogram showed atrial fibrillation rhythm, complete right bundle branch block, and low voltage, which differed from those taken at the original hospitalization (Fig. 2). Although ventricular premature beats originating from the RV had occurred frequently before the aneurysmectomy, we observed few premature ventricular beats. Cardiac ultrasound showed $R V$ dilatation, a giant right atrium (RA), and inferior vena cava dilatation as a result of tricuspid regurgitation due to tricuspid valve leaflet detachment (Fig. 3). Pulmonary hypertension and left ventricular (LV) dysfunction were not observed.

The patient was diagnosed with hepatic coma due to a high plasma ammonia level as well as his consciousness disorder and flapping tremor. An amino-acid preparation was administered intravenously and lactulose was treated by rectal injection. As the RV failure resulted in a worsening of hepatic congestion, his water balance was gradually decreased on hemodialysis. However, we were obliged to discontinue hemodialysis as we could not maintain his blood pressure. The patient died due to progressive anasarca with pleural effusion and ascites that were difficult to control.

Autopsy results showed remarkable thinning of the RV wall, although the LV wall was normal. The myocardium of the RV was extensively replaced by fibrous and fatty tissues, consistent with the original pathology 31 years earlier. The residual

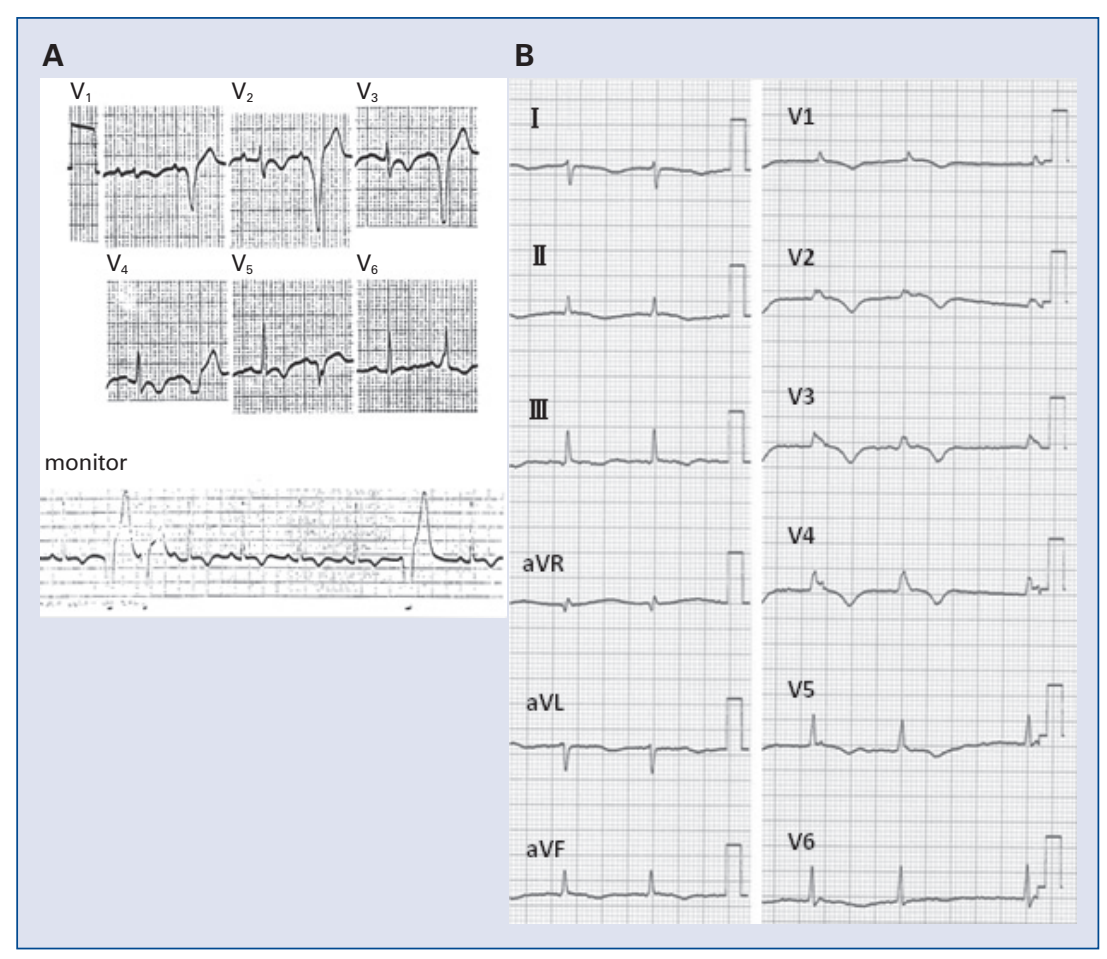

Figure 2. A. Standard electrocardiography and Holter monitor recorded 31 years prior to death; B. Recent electrocardiography showed atrial fibrillation rhythm, complete right bundle branch block, and low voltage. 


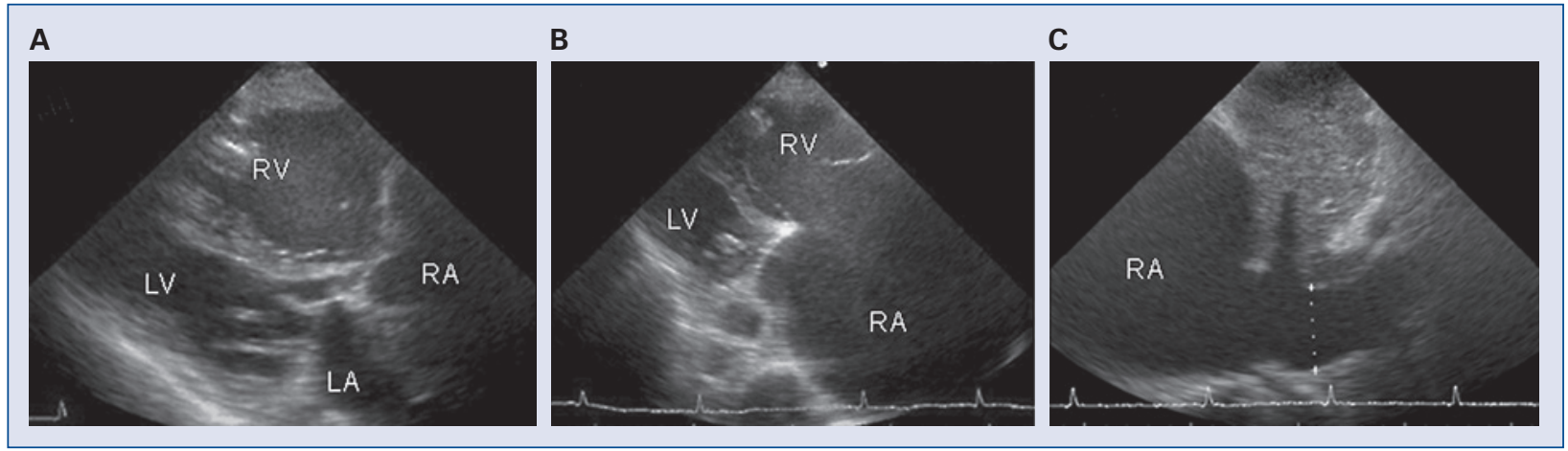

Figure 3. Cardiac ultrasound showed right ventricle (RV) dilatation, a giant right atrium (RA), and inferior vena cava dilatation, although the left ventricle (LV) and left atrium (LA) were normal; A. Parasternal long axis view; B. Parasternal four-chamber view; C. Subcostal view.

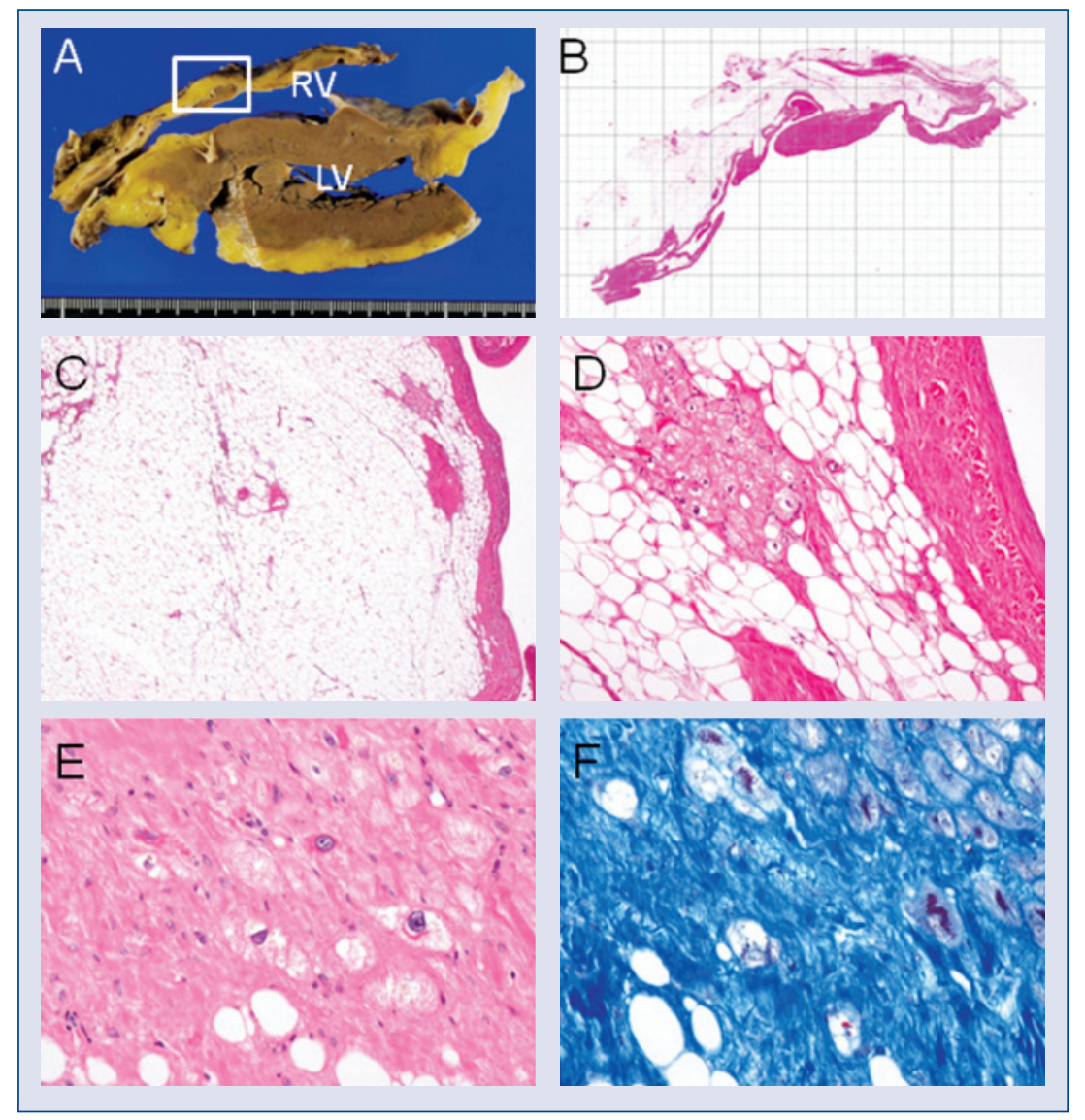

Figure 4. A. Autopsy findings showed marked thinning of the right ventricular (RV) wall, although the left ventricular (LV) wall was normal; B The residual myocardium of the RV was $33.2 \%$ as assessed areas within the white box by magnifying glass; C, D. Cardiomyocytes extensively replaced by fibrous and fatty tissues were observed by low and intermediate power microscopy; E, F. Loss of cardiomyocytes and increase of collagenous fibrous tissues were observed by high power microscopy (E. Hematoxylin-Eosin stain; F. Masson trichrome stain).

$\mathrm{RV}$ myocardium was $33.2 \%$, indicative of residual myocytes $<60 \%$ by morphometric analysis according to the revised Task Force Criteria of ARVC/D
(Fig. 4). Glomerular abnormal changes were rarely observed, whereas portal vein hypertrophy was obvious, suggestive of portal hypertension. 


\section{Discussion}

ARVC/D is predominantly a genetically determined heart muscle disorder associated with ventricular arrhythmias, heart failure, and sudden death $[2,4]$. The main pathologic feature of $A R V C / D$ is the progressive loss of myocardium and its replacement by fibrofatty tissue [5]. In the early stage of the disease, structural changes may be absent or confined to a localized region of the RV. In the present case, the patient originally underwent surgical resection of an RV outflow tract aneurysm that exhibited fatty infiltration. The tissue resected 31 years before consisted almost totally of fatty and fibrous tissue [6]. Pathological autopsy results showed substantial enlargement of the RA and the RV, with fibrofatty replacement, suggesting that the disease had progressed to a more diffuse stage in the RV.

According to the revised Task Force Criteria for the clinical diagnosis of ARVC/D, this case is compatible with a definite diagnosis, as two major criteria and one minor criterion were fulfilled, i.e.: regional $\mathrm{RV}$ akinesia assessed by echocardiography, residual myocytes $<60 \%$ by morphometric analysis with fibrofatty replacement of the RV free wall, and inverted $\mathrm{T}$ waves in leads $\mathrm{V} 1, \mathrm{~V} 2, \mathrm{~V} 3, \mathrm{~V} 4$ and $\mathrm{V} 5$ in the presence of complete right bundle branch block [7].

ARVC/D is considered to be familial with autosomal dominant inheritance, although there are recessive forms associated with a cutaneous phenotype. Genetic variations have been found in the desmosomes responsible for cell-to-cell binding $[2,4]$. However, there have been few studies of familial ARVC/D in genetically heterogeneous populations. Many studies have shown that the natural history of ARVC/D is predominantly related to ventricular arrhythmia and LV involvement [1]. However, there has been no long-term follow-up report of a patient following aneurysmectomy. Although our case originally exhibited several syncopal attacks considered to be induced by ventricular fibrillation, the original RV aneurysmectomy may have contributed to the long-term survival of the patient, as there were no episodes of syncopal attack or lifethreatening ventricular arrhythmia thereafter [6]. Sen-Chowdhry et al. [8] reported LV involvement in $84 \%$ of cases of ARVC using cardiovascular magnetic resonance (CMR) imaging. In the present case, there were no LV abnormalities detected by echocardiography, CMR, or pathological autopsy, which may have contributed to his long-term prognosis. In 15 patients with $\mathrm{ARVC} / \mathrm{D}$ reported by
Blomstrom-Lundqvist et al. [9], three patients were alive at 22,24 , and 28 years, although the mortality rate of this group was $20 \%$. Furthermore, their data suggested that the clinical course of patients with ARVC/D was highly variable and could not be predicted in individual patients.

Unexpectedly, in the present case, pathological evidence of glomerular abnormal changes was rarely observed. As such, pre-renal factor or low cardiac output was considered the cause of chronic renal failure. Repeated RV failure was treated by controlling volume balance on hemodialysis, which may also have contributed to long-term survival.

\section{Conclusions}

The present case suggests that patients with arrhythmogenic RV cardiomyopathy, but without rapid ventricular arrhythmia and LV abnormalities, have a relatively favorable prognosis, although RV dysfunction caused by the extension of muscle may progress.

Conflict of interest: none declared

\section{References}

1. Corrado D, Basso C, Thiene G. Arrhythmogenic right ventricular cardiomyopathy: Diagnosis, prognosis, and treatment. Heart, 2000; 83: 588-595.

2. Syrris P, Ward D, Evans A et al. Arrhythmogenic right ventricular dysplasia/cardiomyopathy associated with mutations in the desmosomal gene desmocollin-2. Am J Hum Genet, 2006; 79: 978-984.

3. Nava A, Bauce B, Basso C et al. Clinical profile and long-term follow-up of 37 families with arrhythmogenic right ventricular cardiomyopathy. J Am Coll Cardiol, 2000; 36: 2226-2233.

4. Corrado D, Basso C, Thiene G. Arrhythmogenic right ventricular cardiomyopathy: An update. Heart, 2009; 95: 766-773.

5. Burke AP, Farb A, Tashko G, Virmani R. Arrhythmogenic right ventricular cardiomyopathy and fatty replacement of the right ventricular myocardium: Are they different diseases? Circulation, 1998; 97: 1571-1580.

6. Kataoka H, Kuroiwa N, Tabuchi H, Ohshige K, Nakamura K, Hashimoto S. Right ventricular aneurysm due to myocardial fatty infiltration: Report of a case. Jpn Cir J, 1982; 46: 1353$-1358$.

7. Marcus FI, McKenna WJ, Sherrill D et al. Diagnosis of arrhythmogenic right ventricular cardiomyopathy/dysplasia: Proposed modification of the Task Force Criteria. Circulation, 2010; 121: 1533-1541.

8. Sen-Chowdhry S, Syrris P, Ward D, Asimaki A, Sevdalis E, McKenna WJ. Clinical and genetic characterization of families with arrhythmogenic right ventricular dysplasia/cardiomyopathy provides novel insights into patterns of disease expression. Circulation, 2007; 115: 1710-1720.

9. Blomstrom-Lundqvist C, Sabel KG, Olsson SB. A long-term follow-up of 15 patients with arrhythmogenic right ventricular dysplasia. Br Heart J, 1987; 58: 477-488. 